INVESTIGACIÓN/RESEARCH

Recibido: 31/10/2014---Aceptado: 25/11/2014---Publicado: 15/12/2014

\title{
GESTIÓN DE LAS REDES SOCIALES TURÍSTICAS EN LA WEB 2.0
}

Trinidad Domínguez Vila: Universidad de Vigo. España. trinidad@uvigo.es Noelia Araújo Vila1: Universidad de Vigo. España. naraujo@uvigo.es

\section{RESUMEN}

Hoy en día es innegable el relevante papel que Internet juega en la sociedad, y el sector turístico no es una excepción. Partiendo de dicha premisa se plantea el estudio de las redes sociales de turismo como elemento clave para la comunicación y comercialización de productos turísticos y las estrategias en que esto puede derivar. Dado el escaso marco teórico existente así como la rápida evolución y cambio de la propia red y su influencia en el sector, se procura establecer un contexto teórico que posibilite el estudio en profundidad de las redes sociales turísticas vinculadas al ecommerce centrado en la tipología business to customer (B2C) ya que son las que ejercen un vínculo entre el usuario y el oferente. Se busca determinar los elementos clave en la oferta de producto y los principales servicios complementarios ofertados por parte de las redes sociales de turismo, así como las estrategias por las que deben optar según su posicionamiento actual.

\section{PALABRAS CLAVE}

Redes sociales - Turismo - Web 2.0 - Comunicación - Estrategia de Comercialización - Internet - E-commerce.

\section{MANAGEMENT OF SOCIAL NETWORKS OF TOURISM IN THE WEB 2.0}

\begin{abstract}
Nowadays, the paper that Internet plays in the society is very important, and the tourist sector is not an exception. Splitting of said premise poses the study of the social networks of tourism as key element for the communication and commercialization of tourist products and their strategies. By cause of the limited theoretical framework existent and the fast evolution and change of the own network

\footnotetext{
${ }^{1}$ Noelia Araújo Vila: Doctora en Dirección y Planificación del Turismo, Máster en Dirección y Gestión de PYMES y Profesora Invitada del Departamento de Organización de Empresas y Marketing de la Universidad de Vigo.
} 
and his influence in the sector, we procures establish a theoretical context to make possible a depth study of the tourist social networks linked to the e-commerce. We have centered our attention in the typology of the e-commerce business to customer (B2C), because they create a relationship between the user and the bidder. It looks to determine the key elements in the offer of product and the main complementary services offered by part of the social networks of tourism, as well as the strategies by which have to opt according to his current positioning.

\section{KEY WORDS}

Social networks - Tourism - Web 2.0 - Communication - Marketing Strategy Internet - E-commerce.

\section{INTRODUCCIÓN: PAPEL DE LAS TICS Y EL SECTOR TURÍSTICO}

La tecnología se ha convertido en un aspecto clave del siglo XXI, siendo en muchos casos un factor relevante en la aparición de nuevos mecanismos o tendencias que hacen frente a las tradicionales. Concretamente se hace referencia a internet, red que se ha convertido en el medio por excelencia para el intercambio de información entre individuos -independientemente de su localización geográfica- (Kahn et. al, 1997:129), $\mathrm{y}$ a todas las herramientas que forman parte de la ya conocida por todos como la red de redes.

El sector turístico, en un intento de hacer frente a las necesidades y carencias de un consumidor exigente y ambicioso, el turista, también ha hecho uso de esta tecnología. El propio turista, conocedor y usuario de las nuevas tecnologías, demanda una serie de servicios de mayor calidad y hace cumplir sus derechos como consumidor. De ahí que el sector turístico oferte sus servicios a través de nuevos mediadores que incrementan el valor añadido y satisfacen las nuevas y actuales demandas.

Según el estudio España Conecta (The Boston Consulting Group, 2011) el impacto de internet en los distintos sectores es evidente, produciendo profundas transformaciones en los mismos, siendo el turismo uno de ellos. Es más, se puede afirmar que el turismo se ha convertido en uno de los campos más afectados por el avance de las tecnologías de la información y comunicación (TICs) (Chamorro, 2008). La forma en que se distribuye la información a través del sector turístico se ha rediseñado, tanto en la forma de planificar como consumir viajes (Buhalis y Law, 2008), lo que ha facilitado el continuo crecimiento del eCommerce en el sector situándose a la cabeza. Según los datos del Observatorio eCommerce (GFK, 2014), más de nueve millones de españoles, un $26,2 \%$ de la población, han comprado algún producto online en los últimos seis meses, siendo el producto más adquirido los viajes, hostelería y ocio con un $10 \%$ del total, donde las reservas de alojamiento $(82 \%)$, el alquiler de vehículos $(80 \%)$ y la compra de billetes de transporte $(74 \%)$ son los de mayor relevancia.

Cifras que avalan tales afirmaciones son el crecimiento en un 8,5\% de las ventas de viajes a través de internet en el año 2010, comportamiento que se debe a la mayor confianza en las transacciones electrónicas percibida por los consumidores y a las 
ofertas y descuentos lanzados a través de la red (DBK, 2011). Aun así, el uso que los consumidores hicieron de internet en 2010 fue principalmente para la búsqueda de información $(96,1 \%)$, -ya que cada vez ésta es mayor y su recopilación y valoración se ha convertido en una práctica habitual para los viajeros (Xiang y Gretzel, 2010)-, y la realización de algún tipo de reserva $(67,2 \%)$, tendencias similares a años anteriores (Instituto de Estudios Turísticos -IET-, 2010). También en el mismo año se dio un incremento del 8\% en las denominadas "agencias de viajes virtuales" (DBK, 2011), aquéllas que dan servicio a usuarios y consumidores y que no necesitan presencia física de un agente para realizar sus compras, estando disponibles en el momento adecuado (Tamayo, 1997).

Internet se convierte por tanto no sólo en fuente de información para el consumidor, sino en un nuevo intermediario con una doble función: distribuidor de productos turísticos y gestor de servicios a empresas relacionadas con el sector turístico. Todo ello se traduce en una evolución de la comercialización turística, haciéndose hincapié en lo inmaterial (información que se transmite rápidamente por la red) y en el reemplazo de intermediarios de la cadena tradicional, agencias de viajes $o$ touroperadores, por páginas web donde el turista encuentra información del servicio y el precio del mismo, de un modo claro, dinámico y transparente (Lanquar, 2001). Internet pasa a ser así un escenario más para la gestión de viajes, convirtiéndose en un medio en crecimiento y de uso habitual para muchos usuarios. La rápida transmisión de la información turística se convierte en algo imprescindible, siendo la solución más eficiente y eficaz internet: acceso a mayor número de canales y/o clientes a un menor coste. De ahí el énfasis de empresas y organismos turísticos en invertir un mayor presupuesto en tecnología e innovación.

En este estudio se pretende determinar la relevancia de las redes sociales turísticas para el sector, sus características y perfiles según producto ofertado a través de alguna de las posibles tipologías de e-commerce. Se busca poder establecer los principales ítems a desenvolver por dichas redes para incrementar su presencia y conseguir un funcionamiento más eficiente y eficaz hacia el usuario potencial y real de productos turísticos, que cada vez más, posee un perfil claramente tecnológico. En resumidas cuentas, se pretende establecer un proceso más nítido para el aprovechamiento de oportunidades generando una ventaja competitiva en relación al resto de agentes del sector.

\section{REDES SOCIALES, COMUNICACIÓN 2.0 Y TURISMO 2.0}

Los avances en las tecnologías web, así como el incremento en el interés de redes sociales, hacen que la industria se vea obligada a reconsiderar formas de planificación y comercialización de productos y servicios turísticos (Di Pietro, Di Virgilio y Pantano, 2012).

Internet juega un nuevo rol como intermediario, superponiéndose a los tradicionales roles de los touroperadores y agencias de viaje, proveyendo a los turistas la posibilidad de comprar numerosos productos y servicios por si mismos (Di Pietro, Di Virgilio y Pantano, 2012). Del mismo modo, las redes sociales ofrecen poderosas herramientas, facilitando numerosos servicios para el mercado turístico, cambiando el rol de los intermediarios tradicionales (Buhalisy Law, 2008; Krachtand Wang, 
2009). Además, los turistas pueden mostrar sus pensamientos y opiniones sobre sus vacaciones y experiencias a través de las mismas, disponibles para toda la comunidad de usuarios de Internet (Dellarocas, 2003).

Las redes sociales pueden jugar diversos papeles dentro del sector turístico: convertirse en vías de comunicación para las empresas y organizaciones, ofertando e intercambiando información; realizar promociones y ventas de productos y servicios (Law y Lau, 2005; Pantano et al., 2011; Tse, 2003); mejorar las funcionalidades de las agencias de viaje y touroperadores tradicionales (Ye et al., 2011); compartir conocimientos (Cheng, 2010), y generar nuevas conexiones entre usuarios, clientes y compañías (Boyd y Ellison, 2007). Todo ello genera que la comunicación a través de redes sociales pueda generar efectos positivos y negativos en las opiniones de los turistas y consecuentemente en sus decisiones (Knights y Willmott, 2007; Pantano et al., 2011).

Las redes sociales poseen poderosas herramientas como la interactividad, la alta calidad, visualización y la rápida búsqueda y transmisión de información, pudiendo convertirse en una de las principales herramientas de elección de destinos turísticos (Hogg, 2010; Kim et al., 2010; Kwon y Wen, 2010).

En definitva, las TICs en el sector turístico han posibilitado además de un mayor conocimiento por parte de los usuarios de la oferta disponible en el sector turístico, precios más flexibles, reducción de los costes de comunicación y distribución, una mayor especialización y diferenciación de servicios, una relación más cercana con el consumidor, redes empresariales más inteligentes, barreras de entrada estables y adquisición de conocimiento (Go, 1992; Buhalis, 1998; Fuchs et al., 2010)

El turismo se está desarrollando muy rápido y adquiriendo mayor relevancia a nivel económico, lo que contribuye al desarrollo laboral, competitividad y sustentabilidad y consecuentemente genera implicaciones sociales, culturales y sobre el propio entorno (Katsoni, 2013b).

Según Werthner y Klein (1999) muchas webs son todavía sólo lugares de información donde no es posible realizar reservas. Se estaría por tanto en una fase 1.0, donde la información es unilateral. En turismo el permanecer en este estado no es posible ni viable. La comunicación bilateral es ya una realidad, y el usuario exige poder participar, interactuar, tanto con la empresa como con otros usuarios.

Las cifras de compras en el sector turístico respaldan esta realidad, en la que el consumidor no sólo se informa de la oferta turística, sino que demanda su consumo a través de la red. Es por ello que en España las compras de productos turísticos representan el 20\% del e-commerce (Estudio comparativo elaborado por Webloyalty en Reino Unido, Francia, España e Italia, 2013). En Italia dicho porcentaje desciende al 14\%, y en Francia no supera el 8\%, convirtiéndose por tanto España en líder de ventas online en lo que a e-turismo respecta. Del mismo modo, el gasto medio en compras a través de internet es inferior a 130 euros en países como Francia y Reino Unido, mientras que en España asciende a 185 euros.

Analizando el lado de la oferta, el $84 \%$ de las empresas del sector de la hostelería y turismo han utilizado alguna vez las redes sociales para promocionar o vender sus productos. Por tanto en España el turismo se posiciona como líder en uso de redes sociales para el e-commerce (Social-Buy, 2012). Las conclusiones de dicho estudio respaldan las fuertes uniones que existen entre las redes sociales y la venta online, 
como que una de cada 10 empresas españolas vende en redes sociales, que Facebook es la red social más utilizada para el social e-commerce $(23,9 \%)$, seguida de Twitter $\left(12^{\prime} 4 \%\right)$, o que casi la mitad de las empresas considera que las redes sociales aportan una ventaja competitiva en la ampliación de los canales de venta (Social-Buy, 2012).

Pero Internet no se ha convertido sólo en un moderno intermediario del canal de distribución turístico o facilitador del e-commerce, sino que realiza un importante papel como herramienta de promoción y comercialización, ofreciendo al sector gran facilidad en la modificación y actualización de la información (Galí, Majó y Vidal, 2000), ya sea a través de la propia página web, blogs, folletos electrónicos, foros, las ya citadas redes sociales y demás integrantes de la web 2.0. Nace así el concepto de Turismo 2.0, Travel 2.0 o e-turismo, definido como la aplicación de la web 2.0 al turismo.

El producto o servicio turístico ya no se entiende como se venía haciendo hasta ahora, sino que es un concepto que está evolucionando, lo cual obliga de un modo simultáneo a un cambio y adaptación por parte de empresas $\mathrm{u}$ organizaciones turísticas, siendo el fenómeno 2.0 y las redes sociales una posibilidad de introducirse y beneficiarse de dichos cambios. A las organizaciones turísticas se les abre una gran oportunidad de crear marca, saber lo que piensan los usuarios, incrementar las ventas gracias a la información, independientemente del tamaño de la empresa -sea una pequeña empresa turística o una empresa líder a nivel nacional-.

Desde el punto de vista de la demanda, a los turistas se les plantean dos alternativas de búsqueda de información en la red: a través de ofertas (vuelos, hoteles, cruceros...), o a través de opiniones de otros usuarios (Sánchez, 2011). La primera alternativa ya no es suficiente, por lo que se ha vuelto habitual en los usuarios el deseo de interacción en la red, acudiendo a comunidades virtuales para ello (Guevara, 2008). El fenómeno Turismo 2.0 o Travel 2.0 abarca infinidad de herramientas de las que el usuario se hace partícipe, desde wikis, foros, blogs, tablones de anuncios a mundos virtuales o vídeos en línea (Schmallegger y Carson, 2008). En dichas comunidades los usuarios perciben satisfacción en el intercambio de información y experiencias, siendo éste el factor más influyente en su uso (Young y Dimitrios, 2008).

Es por ello que dentro del fenómeno 2.0 aplicado al sector turístico, se han abierto paso y penetrado con fuerza las redes sociales. Se está ante herramientas virtuales que "permiten a los usuarios crear un perfil público o semi-público, crear y ver sus propias redes, así como las de otros usuarios, e interactuar con la gente en sus redes" (Subrahmanyaman et al., 2008: 421).

El objetivo de estas redes es acercar e incluso integrar al cliente en dichas comunidades virtuales, (Sobejano, 2009), de tal modo que se creen vínculos que hagan que el propio usuario o cliente se identifique con la empresa y la empresa pueda retroalimentarse de la información y opiniones que éste aporta.

$\mathrm{Su}$ uso se ha convertido en poco tiempo en algo habitual a nivel mundial, siendo los perfiles más activos las mujeres y los jóvenes entre 18 y 34 años (Nielsen, 2011) y el medio de consumo más habitual el ordenador, aunque 2 de cada 5 usuarios ya acceden a las redes sociales a través de sus teléfonos móviles (Nielsen, 2011). Éstas ganan adeptos día a día, siendo la más exitosa y usada a nivel mundial Facebook, con más de 1000 millones de usuarios (Facebook, 2013). Es por ello, que aunque éstas 
surgieron como portales online de encuentro e intercambio de opiniones, vídeos o fotografías entre individuos, fueron muchos los sectores y organizaciones que vislumbraron una oportunidad en las mismas de acercarse y promocionarse ante potenciales clientes. Nacen así los grupos y perfiles de empresas y entidades públicas, entre los que el sector turístico también se ha abierto un hueco. En el caso de Facebook son numerosas las grandes cadenas o empresas hoteleras que poseen perfil y mandan información a sus fans. Pero no sólo las grandes empresas hacen uso de esta red, sino que también lo hacen pequeños hoteles, además de ayuntamientos, agencias de viajes, touroperadores y toda empresa vinculada al sector, ya que es un medio al que un gran número de personas tienen acceso. Además, aunque la tendencia sigue siendo el utilizar estas redes generalistas, porque son a través de las cuales se puede acceder a un mayor número de usuarios, se empiezan a mostrar signos de decantación por otras más verticales y segmentadas (Roca, 2008). Aparecen por tanto redes/comunidades especializadas en el sector turístico, como es el caso de Turismo 2.0, Couchsurfing, TripAdvisor o Tripwolf, denominadas todas ellas redes sociales acotadas al sector turístico, enfocadas a la organización de viajes o al contacto con especialistas de viaje $\mathrm{u}$ otros usuarios viajeros.

\section{REDES SOCIALES PROFESIONALES DEL SECTOR TURÍSTICO}

Las diferentes empresas y sectores del mercado actual, conscientes de la importancia de interactuar con sus consumidores o potenciales consumidores, poco a poco han ido formando parte de las redes sociales de carácter generalista, con la creación de perfiles o grupos (Facebook, Myspace, LynkedIn...). El sector turístico ha hecho uso de las mismas, mas como se acaba de ver en el epígrafe anterior, la web 2.0 y en especial las redes sociales se han convertido en herramientas promocionales habituales, por lo que han aparecido redes sociales específicas para este sector.

Para cualquier destino o empresa turística se hace imprescindible saber gestionarse a través de las redes sociales y relacionarse con sus usuarios o potenciales consumidores. La opinión que éstos tengan, y reflejen posteriormente de dicha empresa, será de gran importante para la toma de decisiones de otros usuarios a la hora de decidir comprar y hacer uso o no de sus servicios. De igual modo la imagen online de un destino turístico es un factor influyente en la decisión de compra por parte de un turista (Di Placido, 2010).

A día de hoy son numerosas las redes sociales a las que el turista puede acceder a la hora de organizar un viaje, desde las que sólo divulgan información de destinos turísticos, hoteles o puntos de interés, hasta las que permiten la interacción más elevada entre usuario y servidor 2.0.

Al hablar de internet, web 2.0 o de modo más específico de redes sociales y el papel que la interactividad juega en este nuevo escenario, se hace referencia a una serie de oportunidades para empresas y organizaciones a la hora de establecer vínculos relacionales con los usuarios (Timmers, 2000). Dentro de estas relaciones destacan (Salgueiro y Sixto, 2009: 173):

- Más intensa captación de la atención del usuario. 
- Mayor conocimiento del perfil del usuario (con el correspondiente uso de un marketing especializado).

- Retroalimentación inmediata.

- Búsqueda de información por parte del propio usuario, denominada información autodirigida.

Volviendo al sector turístico, todas las webs autodenominadas redes sociales se benefician de dichas características, y efectivamente se pueden englobar dentro de esta herramienta, ya que en cierto modo aportan información de ámbito turístico con mayor o menor nivel de interacción con el usuario; pero no todas operan al mismo nivel. Hasta el momento, dada la novedad en el estudio de estas redes profesionales y su reciente aparición y proliferación, no existe una clasificación de las mismas en función de la diferente intensidad del uso de la información e interacción con el usuario. Por ello, en el presente estudio se ha pretendido establecer cierta segmentación o agrupación en función a algún criterio, y analizar así un conjunto homogéneo de las mismas; ya que realmente se está ante un grupo muy heterogéneo en el que establecer comparaciones y análisis se convierte en una tarea complicada. La variable de segmentación escogida es el comportamiento de la web en relación al comercio electrónico o e-commerce. El comercio electrónico, de forma generalizada, consiste en el intercambio de información comercial mediante la transmisión de datos a través de la red, causa origen de la proliferación de empresas y consumidores que contribuyen diariamente a diversificar y transformar esta nueva realidad, creándose diferentes tipos de comercio electrónico para adaptarse a las necesidades del mercado (Montane, 2010), pudiéndose destacar principalmente las agrupaciones que se recogen en la siguiente tabla (Tabla 1).

\begin{tabular}{|c|c|c|}
\hline TIPO & COMPONENTES & CARACTERÍSTICAS \\
\hline $\begin{array}{c}\text { BUSINESS TO } \\
\text { BUSINESS - B2B }\end{array}$ & $\begin{array}{l}\text { Entre empresas que } \\
\text { operan en la red. Se } \\
\text { distinguen } \\
\text { modalidades: } \\
\text { - El mercado } \\
\text { controlado por el } \\
\text { vendedor en busca de } \\
\text { compradores. } \\
\text { - El mercado } \\
\text { controlado por el } \\
\text { comprador que busca } \\
\text { proveedores. } \\
\text { - El mercado } \\
\text { controlado por } \\
\text { intermediarios que } \\
\text { persiguen el acuerdo } \\
\text { entre vendedores v }\end{array}$ & $\begin{array}{l}\text { Para la optimización: } \\
\text { - Experiencia en el mercado } \\
\text { concreto. } \\
\text { - La oferta debe ser un valor } \\
\text { añadido. } \\
\text { - Evitar fallos de producción, } \\
\text { logística y distribución. } \\
\text { Ventajas principales: } \\
\text { - Reducción de costes. } \\
\text { - Ampliación de mercado. } \\
\text { - Aumento de la velocidad. } \\
\text { - Centralización de oferta y } \\
\text { demanda. }\end{array}$ \\
\hline
\end{tabular}




\begin{tabular}{|c|c|c|c|}
\hline & compradores. & \multicolumn{2}{|c|}{$\begin{array}{l}\text { - Información de compradores, } \\
\text { vendedores, productos y precios en } \\
\text { un lugar común. } \\
\text { - Mayor control de las compras }\end{array}$} \\
\hline $\begin{array}{l}\text { CUSTOMER TO } \\
\text { CUSTOMER - C2C }\end{array}$ & 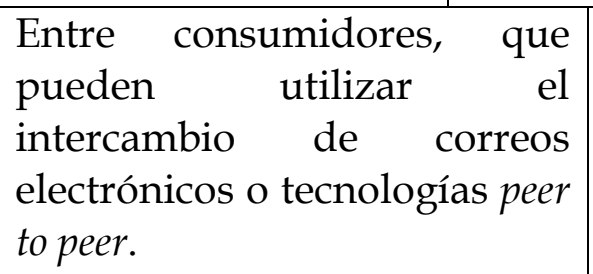 & \multicolumn{2}{|c|}{$\begin{array}{l}\text { Negocio cuyo objetivo es } \\
\text { facilitar la comercialización } \\
\text { de productos y/o servicios } \\
\text { entre particulares. }\end{array}$} \\
\hline $\begin{array}{c}\text { BUSINESS TO } \\
\text { CUSTOMER - B2C }\end{array}$ & \multicolumn{2}{|c|}{$\begin{array}{l}\text { Entre las empresas y el consumidor. } \\
\text { Modalidades más destacadas: } \\
\text { - Intermediarios on-line: Se facilitan las } \\
\text { transacciones entre compradores y } \\
\text { vendedores a cambio de una } \\
\text { contraprestación económica a modo de } \\
\text { porcentaje. } \\
\text { - Modelos basados en la publicidad: } \\
\text { Publicidad basada en el sistema, donde } \\
\text { las empresas tienen sitios web de un } \\
\text { inventario, que venden a las partes } \\
\text { interesadas. } \\
\text { - Modelos basados en la comunidad: } \\
\text { Las empresas facultan a los usuarios } \\
\text { para interactuar en todo el mundo, en } \\
\text { áreas similares de interés. } \\
\text { - Modelos basados en tarifas: Un } \\
\text { sistema de pago basado en el sistema. En } \\
\text { este caso la empresa cobra una tarifa de } \\
\text { suscripción para ver su contenido. }\end{array}$} & $\begin{array}{l}\text { Ventajas } \\
\text { principales: } \\
\text { - Compra más } \\
\text { cómoda y más } \\
\text { rápida. } \\
\text { - Ofertas y } \\
\text { precios siempre } \\
\text { actualizados. } \\
\text { - Centros de } \\
\text { atención al cliente } \\
\text { integrados en la } \\
\text { web. principal } \\
\text { El la } \\
\text { inconveniente } \\
\text { deriva de la } \\
\text { atención que el } \\
\text { consumidor debe } \\
\text { tener en relación a } \\
\text { la seguridad en las } \\
\text { operaciones de } \\
\text { intercambio }\end{array}$ \\
\hline $\begin{array}{c}\text { BUSINESS TO } \\
\text { ADMINISTRATION } \\
\text { - B2A }\end{array}$ & $\begin{array}{ll}\text { Entre } & \text { la } \\
\text { administración y la } \\
\text { empresas (también } \\
\text { puede darse con los } \\
\text { ciudadanos) para la } \\
\text { realización rrán de } \\
\text { trámites } \\
\text { administrativos. }\end{array}$ & $\begin{array}{l}\text { Las principales } \\
\text { empresa: } \\
\text { - Ahorro conside } \\
\text { esfuerzo. } \\
\text { - La posibilidac } \\
\text { formularios y } \\
\text { procedimientos ac } \\
\text { - Disponibilidad }\end{array}$ & $\begin{array}{l}\text { ventajas para la } \\
\text { erable de tiempo y } \\
\text { de descargarse } \\
\text { modelos de los } \\
\text { lministrativos. } \\
\text { las } 24 \text { horas del }\end{array}$ \\
\hline
\end{tabular}




\begin{tabular}{|c|c|c|c|}
\hline & & & $\begin{array}{l}\text { día. } \\
\text { - Información siempre actualizada. }\end{array}$ \\
\hline $\begin{array}{c}\text { BUSINESS TO } \\
\text { EMPLOYEE - B2E }\end{array}$ & $\begin{array}{l}\text { Entre la } \\
\text { empresa con } \\
\text { sus } \\
\text { empleados. }\end{array}$ & \multicolumn{2}{|c|}{$\begin{array}{l}\text { Ventajas más destacadas: } \\
\text { - Menores costes y burocracia. } \\
\text { - Formación en línea. } \\
\text { - Mayor calidad en la información interna. } \\
\text { - Equipos de colaboración en el entorno } \\
\text { web. } \\
\text { - Integración más ágil del profesional en la } \\
\text { empresa. } \\
\text { - Soporte para la gestión. } \\
\text { - Comercio electrónico interno. } \\
\text { - Fidelización del empleado. }\end{array}$} \\
\hline $\begin{array}{l}\text { CITIZEN TO } \\
\text { GOVERNMENT - } \\
\text { C2G }\end{array}$ & $\begin{array}{l}\text { Entre los } \\
\text { consumidores } \\
\text { y el Gobierno } \\
\text { para el } \\
\text { intercambio } \\
\text { telemático. }\end{array}$ & \multicolumn{2}{|c|}{$\begin{array}{l}\text { Algunos de los servicios más habituales son: } \\
\text { - Información. } \\
\text { - Participación del ciudadano. } \\
\text { - Suscripción para la notificación telemática. } \\
\text { - Pago de tasas e impuestos. } \\
\text { - Sugerencias y reclamaciones. } \\
\text { - Entrada y/o salida a través de registro. } \\
\text { - Diversos servicios, como empleo, sanidad } \\
\text { o educación. }\end{array}$} \\
\hline $\begin{array}{l}\text { BUSINESS TO } \\
\text { GOVERNMENT - } \\
\text { B2G }\end{array}$ & \multicolumn{3}{|c|}{ 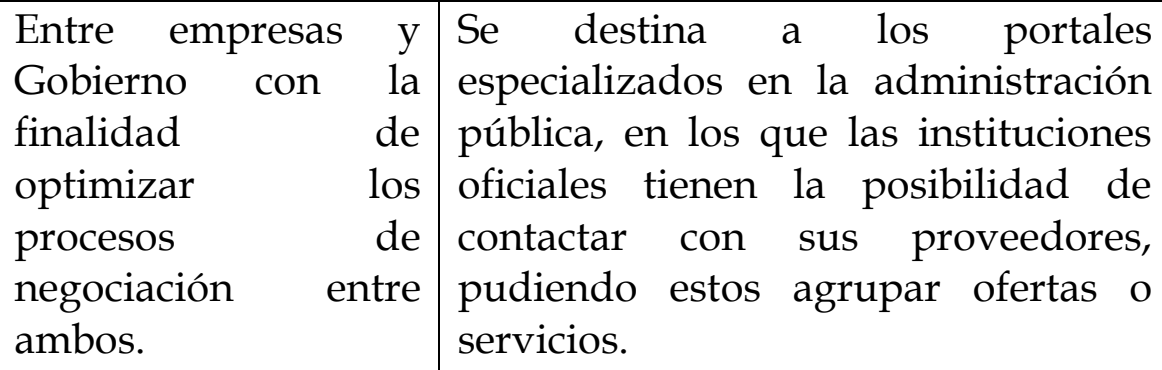 } \\
\hline
\end{tabular}

Tabla 1: Tipologías de comercio electrónico.

Fuente: Elaboración propia a partir de Montane (2010), Valverde (2011) y Asociación

Española de Comercio Electrónico y Marketing Relacional (AECEM) (2009). 
Si lo enumerado en la tabla anterior se vincula al objetivo de la investigación de este trabajo, agrupaciones para estudiar la actividad turística a través de las redes sociales de turismo en internet, se seleccionan tres categorías principalmente, es decir, aquellas en las que existe $e$-commerce directo:

- B2C: la interacción se da desde un agente turístico a un usuario/turista 2.0, dando opción a intercambio de información, registro de usuario, compra y venta tradicional $\mathrm{u}$ oferta diferenciada de información y divulgación de la misma (ejemplo de ello sería Trivago). Se centrará la atención prioritaria en los modelos basados en la comunidad por ser los que más potencian la fidelización entre los agentes.

- B2B: interacción entre agentes turísticos, básicamente información del sector para otros agentes del sector (por ejemplo Turiesfera).

- C2C: interacción entre usuarios/turistas 2.0. Los propios turistas contactan entre sí a través de dichos portales webs, como el caso de Couchsurfing, donde los usuarios de esta red ofrecen sus casas o sus servicios de guía a otros usuarios y viajeros (Ascanio, 2007).

Secundariamente, podrán aparecer redes sociales turísticas que no se puedan englobar en ninguna de las categorías esgrimidas anteriormente, y que se pueden encuadrar dentro de la acepción de Portales webs de información y divulgación, donde tanto agentes turísticos como usuarios/turistas 2.0 pueden dejar información para conocimiento de los demás. Ejemplo de ello es Foursquares, donde se puede acceder tanto a la localización de empresas/organizaciones turísticas como a los lugares visitados por otros turistas.

\section{OBJETIVOS DEL ESTUDIO Y METODOLOGÍA}

Como ya se ha ido desglosando a lo largo del trabajo, se pretende como objetivo fundamental determinar el papel de las redes sociales de turismo para el sector, focalizándose en aquellas que repercutan de forma directa a causa de un intercambio o transacción entre diferentes agentes del sector. Una vez establecidas las webs que cumplan dicho criterio, se analizarán cuáles son las principales características del producto ofertado, así como los servicios complementarios que ofrece, y que por lo tanto, generan un valor añadido e incrementan el valor del producto para el consumidor. Poder establecer dichos ítems facilitará el desarrollo de nuevos productos turísticos a través de la innovación y el enfoque experiencial generado por otros consumidores por medio de la web 2.0.

A la hora de determinar la metodología a emplear se partía de la imposibilidad de acotar la población a estudiar, derivado del problema existente por la falta de un marco teórico estable y profundo sobre qué son y cómo funcionan las redes sociales turísticas, así como la falta de datos secundarios sobre los que poder trabajar. Por todo ello, se optó por realizar un barrido a través de los principales buscadores en internet (ver Tabla 2) y establecer así sus principales características a través de un análisis de contenido. 


\begin{tabular}{|c|c|}
\hline RED SOCIAL & DESCRIPTOR \\
\hline Couchsurfing & $\begin{array}{l}\text { Red social de intercambio o préstamo de alojamiento propio } \\
\text { por parte de usuarios individuales. }\end{array}$ \\
\hline Delicious & Red social de temáticas variadas, entre ellas viajar. \\
\hline Dopplr & $\begin{array}{l}\text { Red social que posibilita la planificación, habla sobre tipos de } \\
\text { viaje y consejos para viajeros. }\end{array}$ \\
\hline $\begin{array}{l}\text { Easy Travel } \\
\text { Connection }\end{array}$ & Red social para profesionales del turismo. \\
\hline Foursquares & $\begin{array}{l}\text { Red social que ofrece un servicio basado en localización web } \\
\text { aplicada a las redes sociales. }\end{array}$ \\
\hline Gogobot & $\begin{array}{l}\text { Red social para búsqueda de hoteles, vacaciones, cosas que } \\
\text { hacer o restaurantes. }\end{array}$ \\
\hline LonelyPlanet & Red centrada en guías de viajes mundial. \\
\hline Los Viajeros & Red social de intercambio de experiencias turísticas. \\
\hline Minube & $\begin{array}{l}\text { Red que da información útil sobre destinos, planificador y } \\
\text { gestor de viajes. }\end{array}$ \\
\hline Touristeye & Red social para planificación de viajes. \\
\hline Travellution & $\begin{array}{l}\text { Red que conecta a viajeros de diferentes partes del mundo que } \\
\text { quieran realizar un viaje en común. }\end{array}$ \\
\hline TripAdvisor & $\begin{array}{l}\text { Red, buscador y gestor de servicios turísticos (alojamiento, } \\
\text { vuelos, etc), todo ello complementado con opiniones de } \\
\text { usuarios. }\end{array}$ \\
\hline Tripatini & $\begin{array}{l}\text { Red donde los usuarios recogen sus viajes, fotos, experiencias } \\
\text { y similares. }\end{array}$ \\
\hline Tripl & $\begin{array}{l}\text { Red que permite la interactuación con otros usuarios o con } \\
\text { locales del destino seleccionado para viajar. }\end{array}$ \\
\hline Trippy & $\begin{array}{l}\text { Red social de intercambio de experiencias entre viajeros y } \\
\text { expertos. }\end{array}$ \\
\hline Tripsay & $\begin{array}{l}\text { Red y buscador de hoteles y realiza recomendaciones de } \\
\text { lugares a los que viajar. }\end{array}$ \\
\hline Tripwolf & Red centrada en proporcionar guías de viajes mundial. \\
\hline Trivago & Red, buscador y gestor de hoteles. \\
\hline
\end{tabular}




\begin{tabular}{|c|l|}
\hline Turiesfera & Red social de profesionales del sector turístico. \\
\hline Turismo 2.0 & Red social de profesionales del sector turístico. \\
\hline Twigmore & $\begin{array}{l}\text { Herramienta de red de viajes en Facebook, permite a los } \\
\text { usuarios aprovechar la red de su amigo para contactos locales. }\end{array}$ \\
\hline Viajaris & $\begin{array}{l}\text { Red que facilita información sobre viajes y muestra ofertas de } \\
\text { paquetes turísticos. }\end{array}$ \\
\hline ViajeRed & Red de blogs y webs de viaje. \\
\hline Viamedius & Red social sobre las experiencias de viaje de los usuarios. \\
\hline Wayn & Red social sobre viajes y estilos de vida. \\
\hline
\end{tabular}

Tabla 2: Redes sociales turísticas más destacadas.

Fuente: elaboración propia.

El total de redes sociales de turismo generales localizadas (se excluyeron aquellas que hacían referencia a nichos, bien geográficamente, por actividad o similares) fueron un total de 20, las cuales fueron agrupadas según la variable de segmentación del tipo de e-commerce realizado, pudiendo establecerse 4 grandes grupos que se muestran en la siguiente tabla (Tabla 3). El estudio fue realizado entre el 2 de febrero y el 13 de marzo de 2012.

\begin{tabular}{|c|c|c|}
\hline TIPO DE & \multicolumn{2}{|l|}{ WEBS } \\
\hline $\begin{array}{c}\text { BUSINESS TO } \\
\text { BUSINESS - B2B }\end{array}$ & $\begin{array}{ll}\text { - } & \text { Easy Travel Connection } \\
\text { - } & \text { Turiesfera }\end{array}$ & - Turismo 2.0 \\
\hline $\begin{array}{l}\text { CUSTOMER TO } \\
\text { CUSTOMER - C2C }\end{array}$ & $\begin{array}{ll}\text { - } & \text { Couchsurfing } \\
\text { - } & \text { Travellution }\end{array}$ & - $\quad$ Tripl \\
\hline $\begin{array}{c}\text { BUSINESS TO } \\
\text { CUSTOMER - B2C }\end{array}$ & $\begin{array}{ll}\text { - } & \text { Dopplr } \\
\text { - } & \text { Minube } \\
\text { - } & \text { TripAdvisor } \\
\text { - } & \text { Tripsay }\end{array}$ & $\begin{array}{ll}\text { - } & \text { Tripwolf } \\
\text { - } & \text { Trivago } \\
\text { - } & \text { Viajaris }\end{array}$ \\
\hline $\begin{array}{l}\text { INFORMACIÓN Y } \\
\text { DIVULGACIÓN }\end{array}$ & $\begin{array}{ll}\text { - } & \text { Delicious } \\
\text { - } & \text { Foursquares } \\
\text { - } & \text { LonelyPlanet } \\
\text { - } & \text { ViajeRed }\end{array}$ & $\begin{array}{ll}\text { - } & \text { Tripatini } \\
\text { - } & \text { Viamedius } \\
\text { - } & \text { Wayn }\end{array}$ \\
\hline
\end{tabular}

Tabla 3: Segmentación de redes sociales turísticas en función de usuarios. Fuente: elaboración propia. 
Dado que el estudio pretende determinar la relevancia de las redes sociales turísticas para la actividad, incidiendo en la importancia para el desarrollo de producto y el intercambio de experiencias, se opta por analizar las web pertenecientes al B2C, ya que se genera una transición entre vendedor y comprador, existe una generación de opiniones sobre los productos basada en las experiencias de los usuarios y son las de mayor repercusión a causa del número de usuarios que poseen. Se desechan las de B2B, al ser muy específicas y sólo vinculantes a los oferentes del sector; las C2C también al non generar directamente intercambios o flujos monetarios; y las de información y divulgación porque no existe proceso de comercialización.

\section{ANÁLISIS Y RESULTADOS}

Una vez analizados los datos, se pueden diferenciar dos tipos de resultados, aquéllos basados en la oferta de productos y los de servicios complementarios ofrecidos. En relación al primero de ellos (Tabla 4), se pueden observar dos variables de análisis, la presencia de cada uno de los productos ofertados en el total de las webs y de forma individualizada en cada una de ellas.

Si se toma el conjunto, destaca que los servicios ofertados con mayor predominio son los referentes a alojamiento, y concretamente los vinculados a la oferta hotelera, ambos con el mismo porcentaje, $85,7 \%$, es decir, que todas las webs que ofertan alojamiento tienen la opción de búsqueda de hoteles, seguida de hostales/pensiones y apartamentos. Los albergues o alquiler de casas representan un dato residual. El siguiente servicio ofertado de forma más notable es la búsqueda de vuelos, con un $42,8 \%$ de presencia en las webs. La restauración tiene una menor presencia, $28,5 \%$ y el resto de servicios ofertados tiene un comportamiento puntual o aislado.

Realizando el mismo análisis pero de forma individualizada, web a web, se deduce que Tripwolf es la web que oferta el mayor número de productos, un 63,6\%, cubriendo los ámbitos de alojamiento, actividades, tours y vuelos. Se considera que debería seguir mejorando e incrementando su implementación a través de la inclusión de la restauración en su oferta de búsqueda, lo cual le posibilitaría afianzar su posicionamiento como líder. TripAdvisor y Minube poseen el mismo porcentaje de servicios ofertados, ambas centrándose principalmente en alojamiento y vuelos. El resto tiene una menor incidencia, así, Tripsay tiene una clara tendencia a alojamiento, al igual que Trivago y Dopplr, las cuales ofertan un menor número de opciones. Destacar por último a Viajaris, que sólo se centra única y exclusivamente en la oferta de paquetes turísticos.

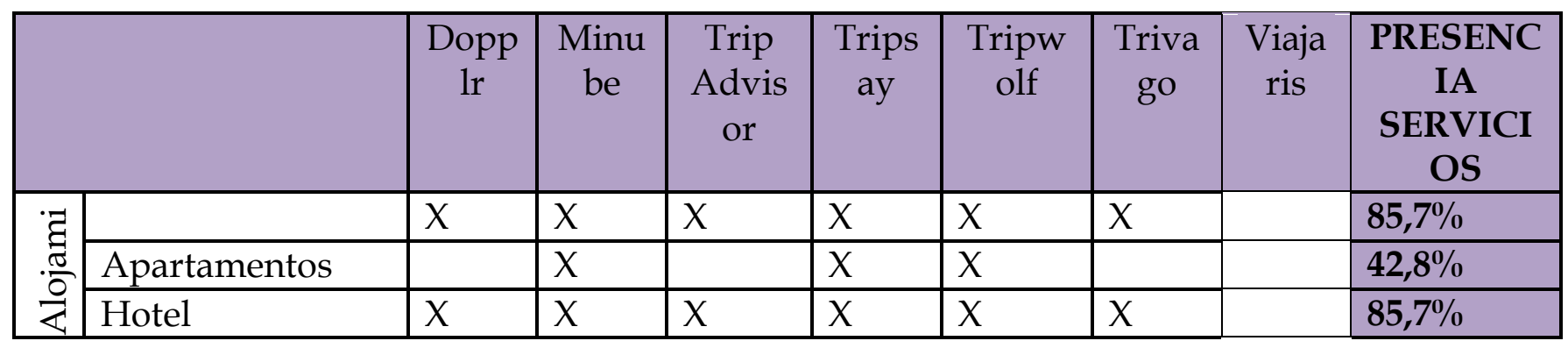




\begin{tabular}{|l|l|l|l|l|l|l|l|l|}
\hline Hostal/pensión & & $\mathrm{X}$ & $\mathrm{X}$ & $\mathrm{X}$ & $\mathrm{X}$ & & & $\mathbf{5 7 , 1} \%$ \\
\hline $\begin{array}{l}\text { Alquiler casa o } \\
\text { similar }\end{array}$ & & & $\mathrm{X}$ & & & & & $\mathbf{1 4 , 2} \%$ \\
\hline Albergue & & $\mathrm{X}$ & & & & & & $\mathbf{1 4 , 2} \%$ \\
\hline Restauración & $\mathrm{X}$ & & $\mathrm{X}$ & & & & & $\mathbf{2 8 , 5} \%$ \\
\hline Actividades & & & & & $\mathrm{X}$ & & & $\mathbf{1 4 , 2} \%$ \\
\hline Tours & & & & & $\mathrm{X}$ & & & $\mathbf{1 4 , 2} \%$ \\
\hline Vuelos & & $\mathrm{X}$ & $\mathrm{X}$ & & $\mathrm{X}$ & & & $\mathbf{4 2 , 8} \%$ \\
\hline Paquetes turísticos & & & & & & & $\mathrm{X}$ & $\mathbf{1 4 , 2} \%$ \\
\hline $\begin{array}{l}\text { TOTAL SERVICIOS } \\
\text { WEB }\end{array}$ & $27,2 \%$ & $54,5 \%$ & $54,5 \%$ & $36,3 \%$ & $63,6 \%$ & $18,1 \%$ & $9 \%$ & \\
\hline
\end{tabular}

Tabla 4: Productos y servicios ofertados de forma individualizada y conjunta por las webs analizadas.

Un elemento clave a tener en cuenta y que incide de forma directa en el análisis e incidencia de las webs analizadas es el número de gestores y buscadores con los que trabajan, ya que no tiene la misma representatividad una web que oferta el $80 \%$ de productos pero con sólo un buscador, que aquélla que oferta un menor número pero con más buscadores. Los resultados son diferenciados tanto en cantidad como en calidad, dado que la comparativa y eficiencia depende del número de salidas. Así, en la tabla 5, se puede advertir que aunque Trivago realiza una menor oferta en tipologías de alojamiento, la búsqueda a través de ella da como resultado un mayor número de salidas ya que posee 23 gestores/buscadores, casi el triple que Tripwolf, que es el que más tipologías de alojamiento oferta.

\begin{tabular}{|l|l|l|l|l|l|l|l|}
\hline & $\begin{array}{c}\text { Dopp } \\
\text { lr }\end{array}$ & $\begin{array}{c}\text { Minu } \\
\text { be }\end{array}$ & $\begin{array}{c}\text { Trip } \\
\text { Advis } \\
\text { or }\end{array}$ & $\begin{array}{c}\text { Trips } \\
\text { ay }\end{array}$ & $\begin{array}{c}\text { Tripw } \\
\text { olf }\end{array}$ & $\begin{array}{c}\text { Triva } \\
\text { go }\end{array}$ & $\begin{array}{c}\text { Viajar } \\
\text { is }\end{array}$ \\
\hline $\begin{array}{l}\mathrm{N}^{\mathrm{o}} \text { de gestores y y } \\
\text { buscadores principales } \\
\text { (total de 25) }\end{array}$ & 2 & 2 & 11 & 1 & 9 & 23 & 2 \\
\hline
\end{tabular}

\section{Tabla 5: Número de gestores/buscadores principales en las Web.}

Este dato es fundamental a la hora de determinar el tipo de web que escoger para ofertar nuestros productos como empresa, o para buscar aquellas ofertas que más se adapten a nuestros perfiles y gustos como clientes potenciales. En definitiva, valorar cantidad de búsquedas o variedad en la tipología de búsqueda. Lo que sí queda claro es que un gestor/buscador básico a introducir en la web es Booking (ver tabla 6), dado que en las webs analizadas está presente en el $71 \%$ de ellas, seguido a una distancia considerable por Destinia, Expedia u Hoteles. Reseñar que a la hora de analizar los gestores/buscadores, muchos de éstos funcionan con su propia página web aparte de vincularse a otras, por lo que su representatividad real en el mercado es mayor.

\begin{tabular}{|l|l|}
\hline GESTORES Y & \% REPRESENTATIVIDAD EN LAS \\
\hline
\end{tabular}




\begin{tabular}{|l|l|}
\hline \multicolumn{1}{|c|}{ BUSCADORES } & \\
\hline Accorhotels & 28,6 \\
\hline Atrápalo & 14,3 \\
\hline Booking.com & 71 \\
\hline Destinia.com & 42,9 \\
\hline eDreams & 28,6 \\
\hline Expedia & 42,9 \\
\hline Hoteles.com & 42,9 \\
\hline Hotusa & 28,6 \\
\hline Hotwire.com & 28,6 \\
\hline Intercontinental & 28,6 \\
\hline Kayak & 14,3 \\
\hline Lastminute.com & 14,3 \\
\hline Logitravel.com & 28,6 \\
\hline Muchoviaje.com & 28,6 \\
\hline Orbitz & 28,6 \\
\hline Prestigia & 14,3 \\
\hline Priceline.com & 14,3 \\
\hline Rumbo.es & 42,9 \\
\hline Splendia & 14,3 \\
\hline Tablet & 28,6 \\
\hline Travelgenio & 28,6 \\
\hline Venere.com & 28,6 \\
\hline Viajes Iberia & 14,3 \\
\hline Vuelo24.es & 28,6 \\
\hline Zagat & 28,6 \\
\hline Tata 6 Gest & \\
\hline
\end{tabular}

Tabla 6: Gestores/buscadores principales en las Web analizadas.

Pero hoy en día el consumidor demanda cada vez productos más completos y si se parte de la premisa de que los usuarios de B2C tienen un claro perfil tecnológico, la oferta de productos a través de Internet debe complementarse con una serie de servicios que posea la web y facilite o implemente la calidad del producto ofertado. Ésta es nuestra segunda variable a analizar.

No se puede olvidar que un elemento clave que determinaba el estudio de web C2B focalizado principalmente en el modelo basado en la comunidad, era que todas ellas debían exigir el darse de alta a los usuarios de forma permanente y que éstos generaran una retroalimentación a través de los comentarios una vez disfrutados los productos. Esto queda corroborado en la siguiente tabla (ver tabla 7) donde se puede observar que las opiniones y la creación de una cuenta propia está presente en todas ellas. Aunque los idiomas también poseen una representatividad absoluta, no es una variable tan definitoria a causa de que internet es global en cualquier ámbito, ya no sólo en la actividad turística, sino en todos los ámbitos (educacional, administración, instituciones, etc.). Las fotos y los foros también tienen una alta representatividad, $71,4 \%$, pero menor a la de recomendaciones, que tiene una presencia en el $85,7 \%$ de las web analizadas. La búsqueda de incrementar el valor percibido por parte de los 
usuarios hace que elementos como los blogs o las aplicaciones vayan adquiriendo cada vez más importancia y elementos como las guías de viajes o planificación/calendarios decrezcan.

El análisis individualizado vuelve a destacar a Tripwolf como una de las webs con mayor oferta de servicios complementarios junto a Minube y Viajaris. TripAdvisor y Dopplr se mantienen en el medio mientras que Trivago y Tripsay con el $42,8 \%$ de servicios complementarios ofertados, se muestran con un claro perfil oferente de producto.

\begin{tabular}{|l|l|l|l|l|l|l|l|l|}
\hline & $\begin{array}{l}\text { Dopp } \\
\text { lr }\end{array}$ & $\begin{array}{l}\text { Minu } \\
\text { be }\end{array}$ & $\begin{array}{l}\text { Trip } \\
\text { Adviso } \\
\text { r }\end{array}$ & $\begin{array}{l}\text { Trips } \\
\text { ay }\end{array}$ & $\begin{array}{l}\text { Tripw } \\
\text { olf }\end{array}$ & $\begin{array}{l}\text { Triva } \\
\text { go }\end{array}$ & $\begin{array}{l}\text { Viajar } \\
\text { is }\end{array}$ & $\begin{array}{l}\text { PRESENCI } \\
\text { A } \\
\text { SERVICIO } \\
\text { S }\end{array}$ \\
\hline Idiomas (n $)$ & $\mathrm{X}(1)$ & $\mathrm{X}(7)$ & $\mathrm{X}(29)$ & $\mathrm{X}(1)$ & $\mathrm{X}(5)$ & $\mathrm{X}(27)$ & $\mathrm{X}(1)$ & $100 \%$ \\
\hline Opiniones & $\mathrm{X}$ & $\mathrm{X}$ & $\mathrm{X}$ & $\mathrm{X}$ & $\mathrm{X}$ & $\mathrm{X}$ & $\mathrm{X}$ & $100 \%$ \\
\hline Fotos usuarios & & $\mathrm{X}$ & $\mathrm{X}$ & & $\mathrm{X}$ & $\mathrm{X}$ & $\mathrm{X}$ & $71,4 \%$ \\
\hline Vídeos & & $\mathrm{X}$ & & & & & $\mathrm{X}$ & $28,5 \%$ \\
\hline Foros & $\mathrm{X}$ & & $\mathrm{X}$ & $\mathrm{X}$ & $\mathrm{X}$ & & $\mathrm{X}$ & $71,4 \%$ \\
\hline Blogs & & & $\mathrm{X}$ & & $\mathrm{X}$ & $\mathrm{X}$ & $\mathrm{X}$ & $57,1 \%$ \\
\hline $\begin{array}{l}\text { Inspiración/Re } \\
\text { come-ndación }\end{array}$ & $\mathrm{X}$ & $\mathrm{X}$ & $\mathrm{X}$ & $\mathrm{X}$ & $\mathrm{X}$ & & $\mathrm{X}$ & $85,7 \%$ \\
\hline Aplicaciones & $\mathrm{X}$ & $\mathrm{X}$ & $\mathrm{X}$ & & $\mathrm{X}$ & & & $57,1 \%$ \\
\hline Enlaces rápidos & & $\mathrm{X}$ & & & & & $\mathrm{X}$ & $28,5 \%$ \\
\hline Cuenta propia & $\mathrm{X}$ & $\mathrm{X}$ & $\mathrm{X}$ & $\mathrm{X}$ & $\mathrm{X}$ & $\mathrm{X}$ & $\mathrm{X}$ & $100 \%$ \\
\hline Encuestas & & & & & & & $\mathrm{X}$ & $14,2 \%$ \\
\hline Guías de viaje & & $\mathrm{X}$ & & & $\mathrm{X}$ & & & $28,5 \%$ \\
\hline $\begin{array}{l}\text { Planificación } \\
\text { viaje } \\
\text { calendario... }\end{array}$ & & $\mathrm{X}$ & & & $\mathrm{X}$ & & & $28,5 \%$ \\
\hline $\begin{array}{l}\text { Contacto con } \\
\text { otros usuarios }\end{array}$ & $\mathrm{X}$ & $\mathrm{X}$ & $\mathrm{X}$ & $\mathrm{X}$ & $\mathrm{X}$ & $\mathrm{X}$ & $\mathrm{X}$ & $100 \%$ \\
\hline $\begin{array}{l}\text { TOTAL } \\
\text { SERVICIOS } \\
\text { WEB }\end{array}$ & $50 \%$ & $78,5 \%$ & $64,2 \%$ & $42,8 \%$ & $78,5 \%$ & $42,8 \%$ & $78,5 \%$ & \\
\hline
\end{tabular}

Tabla 7: Servicios complementarios disponibles de forma individualizada y conjunta por las webs analizadas.

\section{CONCLUSIONES}

Es obvia la relevancia de internet en todos los sectores de la economía hoy en día, incluido el sector turístico, ya no sólo en términos de promoción y comunicación de productos y servicios o en información de destinos, sino ya como canal de negocio entre empresa y usuario. Uno de los elementos principales en dicho canal, el B2C, es la web 2.0, que a través de la interactuación entre ambos agentes permite el incremento de valor y percepción de calidad sobre el producto ofertado. Dicha 
comunicación facilita e impulsa la gestión de compra de productos turísticos a través de la red, sin intermediarios, con un menor coste y mayor conocimiento sobre el mismo. Esta inminente tendencia del sector turístico hacia internet desemboca en una falta de teorización fundamentada que posibilite establecer los parámetros y conceptos básicos de estudio. Reflejo de este problema es la falta de una definición clara de red social de turismo desde una perspectiva cibernética, existiendo una gran variedad y amplitud de webs muy diferenciadas pero que se engloban todas ellas dentro de dicho término.

A través de este estudio se puede confirmar que las webs turísticas 2.0 que operan principalmente en mercados tipo B2C, deben cumplir dos premisas básicas, ofertar algún producto turístico y que el usuario deba poseer una cuenta propia y pueda realizar opiniones sobre los productos ofertados, esto es, deben tender hacia modelos basados en la comunidad que posibiliten una mayor fidelización y compromiso entre los agentes. Estos elementos son clave para poder diferenciarse del resto de webs que pueden operar de gestoras o buscadores pero que no implican una vinculación tan directa con el usuario. Es decir, éste no tiene por qué poseer una cuenta propia para acceder a buscar o comprar productos y no es obligatorio que existan opiniones sobre los productos ofertados.

Una vez determinadas las características básicas de las redes sociales turísticas 2.0 encuadradas en el B2C del sector turístico, se ha realizado un análisis de las mismas en base a la oferta de productos y a los servicios complementarios que ofertan. Se deduce que la oferta de alojamiento es el elemento básico, pero éste debe ser complementado con vuelos, restauración o actividades principalmente, y así conseguir un producto ofertado aumentado. En lo relacionado con los servicios prestados, se debe tender a una mayor especialización a través de aplicaciones para otros dispositivos como smartphones o tablets, así como buscar técnicas o canales para implementar las recomendaciones de usuarios porque permiten ampliar la temática y contenidos de la web así como afianzar la lealtad.

Según el análisis realizado se pueden establecer dos claras estrategias: estrategia basada en la amplitud de la oferta de productos y servicios complementarios, o una estrategia centrada en la focalización de la oferta de un producto clave. Así, se muestra el caso de Tripwolf, que es la web que mejor posicionamiento tiene en base al número de productos ofertados y servicios ofrecidos pero con la gran limitación de que el número de buscadores empleado no es muy alto, un total de 9. Dicha característica limita el número de ofertas, que incide directamente en la variedad, tipología y eficiencia comparativa de las mismas para el usuario. En el otro lado está el caso de Trivago, que ha optado por centrar su oferta en un solo producto, alojamiento hotelero, que se considera el de mayor incidencia de todos los analizados. La posible desventaja que posee al especializarse es compensada con el gran número de ofertas que puede obtener, dado que emplea más de 20 buscadores. Estos dos ejemplos citados muestran claramente el escenario actual de las redes sociales de turismo en la web 2.0. La estrategia de mayor relevancia es una estrategia de diferenciación basada en la diversificación de productos ofertados, o en la especialización en alguno de ellos. Si se opta por la primera posibilidad y se desea implementar el posicionamiento en comparación al resto de competidores, una ventaja clara es apostar por incrementar el número de ofertas mediante el aumento 
del número y calidad de los buscadores. Si la escogida es la segunda opción, se debe tender a intentar ya no sólo abarcar un alto número de buscadores, sino que estos sean de alta calidad y prestigio.

Para finalizar, se cree importante destacar que las webs analizadas deben esforzarse en mantener un equilibrio que posibilite la información e interactuación del usuario, así como la búsqueda de productos interesantes para el mismo. En dicho equilibrio debe residir la clave.

\section{REFERENCIAS}

Asociación Española de Comercio Electrónico y Marketing Relacional (AECEM) (2009). Libro Blanco del Comercio Electrónico. Guía Práctica de Comercio Electrónico para PYMES. Madrid: Ministerio de Industria, Turismo y Comercio y AECEM.

Ascanio, A. (2007). Proyecto CouchSurfing. Una red de intercambio de viajeros. PASOS, 5 (3), pp. 407-408.

Boyd, D. \& Ellison, N.B. (2007). Social Network Sites: Definition, History and Scholarship. Journal of Computer-Mediated Communication, 13 (1), pp. 210-230. doi: 10.1111/j.1083-6101.2007.00393.x

Buhalis, D. (1998). Tourism and Information technologies: Past, present and future. Tourism Recreation Research, 25 (1), pp. 41-58.

Buhalis, D. \& Law, R. (2008). Progress in information technology and tourism management: 20 years on and 10 years after the Internet-the state of eTourism research. Tourism Management, 29 (4), pp. 609-623. doi: 10.1016 / j.tourman.2008.01.005

Chamorro, R. (2008). Una revolución en el turismo gracias a las TIC. BIT, 170, pp. 3033.

Cheng, H. (2010). Seeking knowledge or gaining legitimacy? Role of social networks on new practice adoption by OEM suppliers. Journal of Business Research, 63 (8), pp. 824-831. doi: 10.1016/j.jbusres.2009.09.023

Dellarocas, C. (2003). The digitization of word of mouth, promise and challenges of online feedback mechanisms. Management Science, 49 (10), pp. 1407-1424. doi: http:/ / dx.doi.org/10.1287/mnsc.49.10.1407.17308

DBK (2011). Informe especial de DBK: Comercio electrónico de Viajes. DBK informa, análisis de sectores. VIII Congreso Turismo y Tecnologías de la Información y las Comunicaciones Turitec 2010. 
Di Placido, A. (2010). Interactividad usuario-usuario y redes sociales online en el sector turístico. Análisis de las páginas web turísticas oficiales de las administraciones andaluzas.

Di Pietro, L., Di Virgilio, F. \& Pantano, E. (2012). Social network for the choice of tourist destination: attitude and behavioural intention. Journal of Hospitality and Tourism Technology, 3 (1), pp. 60-76. doi: 10.1108/17579881211206543

Fuchs, M., Höpken, W., Föger, A. \& Kunz, M. (2010). E-business readiness, intensity and impact: an Austrian destination management organization study. Journal of Travel Research, 49 (2), pp. 165-178. doi: 10.1177 / 0047287509336469

Galí, N., Majó, J. \& Vidal, D. (2000). Patrimonio Cultural y Turismo: Nuevos modelos de promoción vía Internet. Cuadernos de Turismo, 6, pp. 73-87.

GFK (2014). Digital Marke Intelligence. Observatorio eCommerce.

Go, F. (1992). The role of computerised reservation system in the hospitality industry. Tourism Management, 13 (1), pp. 22-26.

Guevara, A. (2008). La web 2.0 y su aplicación al sector turístico. Travel 2.0. BIT, 170, pp. 38-40.

Hogg, T. (2010). Inferring preference correlations from social networks. Electronic Commerce Research and Applications, 9 (1), pp. 29-37. doi: 10.1016 / j.elerap.2009.04.006

Instituto de Estudios Turísticos (IET) (2010). Encuesta de Movimientos Turísticos de los Españoles (Familitur). Madrid: Ministerio de Industria, Turismo y Comercio.

Kahn, R., Leiner, B.M., Cerd, V., Clark, D., Kleinrock, L., Lynch, D., Postel, J., Roberts, L. \& Wolff, S. (1997). The Evolution of the Internet as a Global Information System. Intl. Inform \& Libr. Rev, 29, pp. 129-151. doi: 10.1006/iilr.1997.0042

Katsoni, V. Giaoutzi, M. \& Nijkamp, P. (2013). Market segmentation in tourism - An operational assessment Framwork. Working Paper, Research Memorandum, 2013-21. Amsterdam: Faculty of Economics and Business Administration.

Kim, W., Jeong, O.-R. \& Lee, S.-W. (2010). On social web sites. Information Systems, 35 (2), pp. 215-236.

Knights, D. \& Willmott, H. (2007). Introducing Organizational Behavior Management. Thomson Learning, London. 
Kracht, J. \& Wang, Y. (2009). Examining the tourism distribution channel: evolution and transformation. International Journal of Contemporary Hospitality Management, 22 (5), pp. 736-757. doi: 10.1108 / 09596111011053837

Kwon, O. \& Wen, Y. (2010). An empirical study of the factors affecting social network service use. Computers in Human Behaviour, 26, pp. 254-263. doi: 10.1016/ j.chb.2009.04.011

Lanquar, R. (2001). Marketing turístico. Barcelona: Ariel Turismo, Colección AECIT.

Law, R. and Lau, W. (2005). A study of the perceptions of Hong Kong hotel managers on the potential disintermediation of travel agencies. Journal of Travel and Tourism Marketing, 17 (3), pp. 117-131. doi: 10.1300/J073v17n02_10

Montane, J. (2010). Clases de comercio electrónico: B2B, B2C, B2A, B2E, C2C, C2G, B2G. Economía y Empleo.

Nielsen (2011). State of Media: The Social Media Report. Recuperado de http://blog.nielsen.com/nielsenwire/social. Consultado el 18/02/2013.

Pantano, E., Servidio, R. \& Viassone, M. (2011). An exploratory study of the touristcomputer interaction: the role of web site usability on hotel quality perception. International Journal of Digital Content Technology and its Applications, 5 (5), pp. 208216.

Roca, G. (2008). Soluciones para nuevas realidades. Modelos de negocio en las redes sociales. TELOS, 76.

Salgueiro, M. \& Sixto, J. (2009). Os blogs como técnica de marketing 2.0 para as empresas informativas. Observatorio (OBS) Journal, 11, pp. 170-186.

Sánchez, E. (2011). El turismo 2.0. Un nuevo modelo de promoción turística. REDMARKA, 6, pp. 33-56.

Schmallegger, D. \& Carson, D. (2008). Blogs in tourism: changing approaches to information exchange. Journal of Vacation Marketing, 14 (2), pp. 99-110. doi: $10.1177 / 1356766707087519$

Sobejano, J. (2009). Cómo introducir la Web 2.0 en el trabajo. Comunidad Hosteltur. Online en: http://www.hosteltur.com/59047_como-introducir-web-20trabajo.html

Social-Buy (2012). I Barómetro del Social E-commerce en España, 2012.

Subrahmanyam, K., Reich, S.M., Waechter, N. \& Espinoza, G. (2008). Online and offline social Networks: use of social networking sites by emerging adults. Journal 
of applied development psychology, 29 (6), pp. 420-433. doi: 10.1016/ j.appdev.2008.07.003

Tamayo, B. (1997). Las agencias de viajes frente a las nuevas tecnologías de distribución turística. Fundación COTEC.

The Boston Consulting Group (2011). España conecta. Cómo transforma internet la economía española. Informe independiente de BCC encargado por Google.

Timmers, P. (2000). Strategies and models for business-to-business trading electronic commerce. United Kingdom: John Wiley and sons.

Tse, A.C. (2003). Disintermediation of travel agents in the hotel industry. Hospitality Management, 22 (4), pp. 453-460. doi: 10.1016 / S0278-4319 (03) 00049-5

Xiang, Z. \& Gretzel, U. (2010). Role of social media in online travel information search. Tourism Management, 31 (2), pp. 179-188. doi: 10.1016/ j.tourman.2009.02.016

Ye, Q., Law, R., Gu, B. and Chen, W. (2011). The influence of user-generated content on traveller behavior: an empirical investigation on the effects of e-word-of mouth to hotel online booking. Computers in Human Behavior, 27 (2), pp. 634-639. doi: 10.1016 / j.chb.2010.04.014

Young, J. \& Dimitrios, B. (2008). Web 2.0: a study of online travel community. In P. O'Connor, W. Höpken, U. Gretzel (eds), Information and Communication Technologies in Tourism 2008, pp 70-81.

Valverde, M. (2011). Comercio electrónico (E-Commerce). Universidad Hispanoamericana, publicado 7 de Julio de 2011. Recuperado de http://es.scribd.com/doc/60117925/Comercio-Electronico. Consultado el $15 / 02 / 2013$.

Webloyalty (2013). Estudio comparativo en Reino Unido, Francia, España Italia.

Werthner, H. \& Klein, S. (1999). ICT and the changing landscape of global tourism distribution. Electronic Markets, 9 (4), pp. 256-262.

Recursos electrónicos:

Facebook: facebook.es [12/03/2014] 


\section{AUTORAS:}

\section{Trinidad Domínguez Vila:}

Doctora en Gestión y Dirección de Empresas, Máster en Dirección y Planificación del Turismo y Profesora Ayudante Doctora del Departamento de Organización de Empresas y Marketing de la Universidad de Vigo.

\section{Noelia Araújo Vila:}

Doctora en Dirección y Planificación del Turismo y Licenciada en Administración y Dirección de Empresas. Desde 2007 colabora en las tareas de coordinación del Master Universitario en Dirección y Planificación del Turismo de la Universidad de Vigo, imparte clases como profesora invitada en el Departamento de Organización de Empresas y Marketing y coopera en proyectos de investigación del campo turístico y empresarial. Posee un Master en Gestión y Dirección de PYMES por la misma con premio al mejor proyecto empresarial. 